\title{
Modelo de decisión para determinar fuentes de retorno, incentivos y capacidad en un sistema de remanufactura
}

Decision model to determine the return sources, incentives, and capacity in a remanufacturing system

\author{
Alex J. Ruiz-Torres ${ }^{1}$, Daniel Jurburg ${ }^{2}$, Yarlier López-Correa ${ }^{3}$
}

Resumen.- Esta investigación propone un modelo de decisión para gestionar la compra de componentes nuevos y usados en un sistema de manufactura. Los componentes nuevos se adquieren de un proveedor el cual ofrece descuentos que dependen de la capacidad reservada. Los componentes usados provienen de varias fuentes, donde la cantidad que se recibirá de cada fuente depende del incentivo suministrado. El modelo considera dos elementos estocásticos, el nivel de retorno por fuente (para componentes usados) y la posibilidad de que no se reciba el envío del proveedor de componentes nuevos. Se realiza un análisis de sensibilidad basándose en un ejemplo numérico. Los resultados demuestran relaciones significativas entre los costos de operación de las fuentes de retorno, la probabilidad de retornos bajos y el número de fuentes usados, y el nivel de incentivo asignado por fuente. Los experimentos también indican como los incentivos y la capacidad reservada dependen de la probabilidad de que no se reciban las unidades nuevas. La aplicación en la práctica del modelo y de los resultados apoyaría a que las empresas con sistemas de remanufactura de componentes seleccionen el mejor nivel de incentivos a ofrecer, determinar su capacidad y decidir cuales fuentes de retornos usar de manera minimicen sus costos totales.

Palabras clave: Cadena de suministro; ciclo cerrado; logística inversa; incentivos; remanufactura; proveedores.

Summary.- This research proposes a decision model to manage the purchase of new and used components in a re-manufacturing system. New components are purchased from a supplier who offers discounts based on the reserved capacity. The used components comes from various sources, where the quantity to be received from each source depends on the incentive provided. The model considers two stochastic elements: the return level per source (for used components) and the possibility that the shipment of new components is not received. A sensitivity analysis is performed based on a numerical example. The results demonstrate significant relationships between the costs to operate the return sources, the probability of low returns, and the incentive levels assigned to each source. The experiments also indicate how the incentives and the reserved capacity depends on the probability that the new components are not received.

Keywords: Supply chain management; closed loop; reverse logistics; incentives; remanufacturing; suppliers.

\footnotetext{
${ }^{1}$ Facultad de Adm. de empresas, Universidad de Puerto Rico, alex.ruiztorres@upr.edu, ORCID iD: 0000-0002-7528-236X

${ }^{2}$ Facultad de Ing., Universidad de Montevideo, djurburg1@um.edu.uy, ORCID iD: 0000-0001-9226-1520

${ }^{3}$ Facultad de Adm. de empresas, Universidad de Puerto Rico, yarlier.lopez@upr.edu, ORCID iD: 0000-0002-2895-897X
}
Memoria Investigaciones en Ingeniería, núm. 19 (2020). pp 2-24
https://doi.org/10.36561/ING.19.2

SSN 2301-1092 • ISSN (en línea) 2301-1106 
Introducción.- Estudios sobre la logística inversa y las cadenas de suministro de ciclo cerrado (CSCC) son cada día más relevantes [1]. Esta relevancia surge a partir del constante progreso tecnológico, el cual resulta en que muchos productos tengan ciclos de vida más cortos (por ejemplo, copiadoras, automóviles, dispositivos médicos, equipos de construcción, computadoras, teléfonos celulares, etc.), lo cual conlleva en un número cada vez mayor de productos desechados. Los estudios enfocados en la logística inversa y las CSCC son críticos para la sustentabilidad de nuestro medio ambiente debido a que permiten el diseño de sistemas para la recuperación de productos desechados de manera que puedan ser "aprovechados"; por ejemplo, para reciclaje de materiales, reúso de sus componentes, o reparación y reventa del producto.

La cantidad y la calidad de los retornos es difícil de predecir y controlar [2-4]. Múltiples investigaciones indican que la incertidumbre asociada con los retornos es una de las principales características de las redes logísticas con recuperación de productos [5-7]. Esta incertidumbre requiere que los sistemas de CSCC dependan de una combinación de flujos de materiales usados, el llamado flujo inverso, y de materiales nuevos provenientes de proveedores tradicionales [8-10]. Es importante notar que la incertidumbre en los sistemas logísticos no está solamente relacionada a los productos retornados, pero también a posibles fallas de los proveedores de productos nuevos [11].

Los incentivos financieros son utilizados para incrementar los niveles de retornos en una gran variedad de productos [12-14]. El proveer incentivos hace más atractivo el retornar productos, sea pagando por cada producto retornado o proveyendo descuentos para reemplazos [15]. Es claro que para que los incentivos sean de beneficio al sistema, los ahorros asociados con los productos retornados tienen que sobrepasar los gastos de estos incentivos. La cantidad de retornos también está asociada con el sistema de recolección en sí mismo y el número de fuentes de retorno o acopio [16]. Cuanto mayor sea el número de fuentes y puntos de acopio mayor será la cantidad de retornos, pero de igual manera, los costos fijos y operacionales aumentarán.

En esta investigación se propone un modelo para la toma de decisiones en un sistema de manufactura donde se utilizan componentes nuevos y usados. La decisión asociada con los componentes nuevos es la capacidad (cantidad de unidades) a reservar del proveedor. El costo de reservación por unidad depende de la capacidad total reservada, donde hay un descuento por reservar mayores cantidades. Durante cada ciclo, la cantidad adquirida del proveedor tiene que ser menor o igual a la cantidad reservada. Las decisiones asociadas con los componentes usados son acerca de cuáles fuentes serán utilizadas y el incentivo provisto a cada fuente. Cada fuente tiene características diferentes en términos de las posibles cantidades a ser retornadas y los costos de los incentivos.

El modelo considera dos elementos estocásticos. El primero establece que existe la probabilidad en cada ciclo de que no se reciban las unidades requeridas al proveedor, sea por razones internas como la falla de equipo crítico o un problema de calidad, o causas externas como un desastre natural, una huelga en los muelles que manejan el embarque, etc. El segundo establece que la cantidad recibida de cada fuente de retornos varía. Se asume que cada fuente de retorno tiene múltiples niveles de retorno, donde la cantidad recibida y la probabilidad de estos niveles está relacionada con los incentivos. La combinación de los niveles de retorno resulta en un conjunto de posibles escenarios, cada uno con diferentes cantidades de retornos por fuente. En cada escenario se considera ambas situaciones en relación con las unidades nuevas, si se reciben o no.

Durante cada escenario se determina la cantidad recibida de cada fuente de retorno, esto representa al total de unidades usadas disponibles para remanufactura y reúso. El modelo asume que la demanda por ciclo siempre es mayor que este total, por lo que nunca quedaría inventario de 
unidades usadas para un ciclo futuro. Además, esto significa que en todos los ciclos es necesario comprar unidades nuevas (limitado por la cantidad reservada). Además, en el caso de que la suma del total de unidades usadas disponibles y los nuevas compradas al proveedor sea menor que la demanda, se incurre en un costo de pérdida. Este costo se relaciona con no poder cumplir con la demanda, lo que resulta en pérdida de ganancias o pérdida de clientes.

El modelo considera seis costos, dos costos fijos y cuatro costos variables que dependen de los diferentes escenarios de devolución de las fuentes de retorno. Los dos costos fijos son el costo de reservar capacidad para el proveedor y el costo asociado con cuales fuentes de retornos se utilizan. Los cuatro costos variables son el costo de remanufactura, el costo de incentivos, el costo de unidades nuevas adquiridas, y el costo de pérdidas. El objetivo es la minimización de los costos totales y se utiliza un árbol de decisión para determinar los costos totales esperados en función de todos los escenarios de devolución posibles.

Esta investigación expande el trabajo de Ruiz-Torres et al. [17] considerando los siguientes elementos: a) múltiples fuentes de retorno y la decisión sobre qué fuentes de retorno utilizar, b) cada fuente de retorno tiene cantidades de devolución aleatoria, c) la decisión sobre qué capacidad reservar considerando descuentos, d) incertidumbre en la entrega del proveedor, e) incentivos que tienen un efecto en la cantidad de devolución por fuente de retorno y f) costos de pérdida al no poder satisfacer la demanda. Los elementos incluidos en esta versión son relevantes porque sirven para caracterizar diferentes sistemas asociados con el retorno de componentes, en particular cuáles fuentes de retorno utilizar. Esto representa por ejemplo áreas geográficas donde se recogería el producto usado, o tipos de productos donde se utiliza el componente.

El resto de este artículo está organizado de la siguiente manera. La Sección 2 examina la literatura pertinente y la sección 3 presenta el modelo propuesto incluyendo un ejemplo numérico. La sección 4 presenta un análisis de sensibilidad. Por último, la Sección 5 proporciona conclusiones e indicaciones para futuras investigaciones.

2. Revisión de la literatura.- La revisión de literatura se enfoca en dos temas principales que sirven de base para el modelo propuesto y el análisis de esta investigación. Estos dos temas son los modelos de redes de CSCC y los modelos de selección y asignación de órdenes a proveedores.

2.1. Modelo de redes CSCC.- Existe una gran variedad de investigaciones relacionadas con el diseño de redes CSCC y los modelos de logística inversa. Entre los autores que han estudiado y analizado la literatura sobre el diseño de redes y los modelos de logística inversa se encuentran Govidan y Soleimani [1], Coenen et al. [4], Govindan et al. [18], Braz et al. [19] y De Giovanni y Zaccour [20]. Todas estas revisiones de literatura caracterizan estudios anteriores para determinar áreas de relevancia y factores importantes, además de proponer temas donde hacen falta más investigaciones.

Múltiples autores han estudiado la incertidumbre en la demanda, los retornos y la calidad de los retornos. Pishvaee et al. [2] propusieron un modelo de programación lineal para una CSCC considerando un solo período y con etapas múltiples que aborda las actividades de recuperación y eliminación para gestionar las incertidumbres con respecto a la cantidad y calidad de los productos retornados. El-Sayed et al. [21] propusieron una red de CSCC de varios períodos y múltiples niveles considerando la demanda incierta de los clientes y las devoluciones de productos. Zeballos et al. [22] desarrollaron una formulación MILP ("Mixed Integer Linear Program") de dos etapas para una red CSCC que aborda simultáneamente las decisiones de diseño y planificación en un sistema con cantidades y calidad inciertas. Benedito y Corominas [3] desarrollaron un modelo donde las tasas de retorno dependen de la demanda, el ciclo de vida del producto y la tasa de 
retorno. Cardoso et al. [24] propuso un modelo MILP para abordar las decisiones de diseño y planificación teniendo en cuenta simultáneamente las actividades de producción, distribución y logística inversa bajo la condición de incertidumbre de la demanda. Zeballos et al. [25] presentaron una formulación para abordar una red general de CSCC de múltiples períodos y múltiples productos al considerar las incertidumbres de los proveedores de materias primas y la demanda de los clientes. Khatami et al. [26] desarrollaron una formulación de dos etapas para abordar una red CSCC de varios períodos y múltiples productos bajo incertidumbre en las demandas de los productos y la cantidad de productos retornados. Giri y Sharma [27] consideraron la incertidumbre en una red CSCC como porcentaje de productos usados que contienen partes útiles, así como la probabilidad de que uno de sus dos nuevos proveedores de componentes no entreguen productos. Keyvanshokooh et al. [28] desarrollaron un modelo de programación estocástica híbrida que considera escenarios estocásticos con respecto a los costos de transporte, las demandas y las devoluciones de productos.

Hosoda y Disney [29] desarrollaron políticas de optimización en un CSCC con tasas variables de la demanda y de los retornos. Zeballos et al. [30] propusieron un modelo MILP estocástico de dos etapas para una red CSCC considerando las incertidumbres en la cantidad y calidad de los retornos. Wu et al. [31] consideraron la demanda incierta de productos, los costos de operar las instalaciones y las unidades de productos reciclados mientras que Kim et al. [32] abordaron las incertidumbres en la demanda y los retornos de una red CSCC en la industria de la moda. Ruiz-Torres et al. [17] consideraron múltiples escenarios para las devoluciones y la condición de las devoluciones en un modelo CSCC donde la entrega de nuevos componentes también es incierta. Ponte et al. [33] investigaron la dinámica de un sistema híbrido de fabricación / remanufactura considerando la incertidumbre en el volumen de retorno y concluyen que existe una relación significativa entre el rendimiento operativo y esta incertidumbre.

Los estudios de Masoudipour et al. [7], Taleizadeh et al. [10], Ruiz-Torres et al. [17], De Giovanni y Zaccour [20], Aras y Aksen [34], Aras et al. [35], He [36], De Giovanni et al. [37], Bhattacharya et al. [38], y Modak et al. [39] han examinado cómo los incentivos impactan la cantidad y la calidad de los artículos retornados. Aras y Aksen [34] y Aras et al. [35] propusieron modelos mixtos de ubicación-asignación de instalaciones para establecer los valores y ubicaciones óptimos de los incentivos. He [36] investigó una red CSCC donde la recuperación del componente es estocástica y depende de los incentivos ofrecidos para maximizar las ganancias totales. De Giovanni et al. [37] investigaron una red CSCC que consta de un fabricante y un minorista, donde ambos participan en un programa de recuperación de productos, ofreciendo incentivos a los consumidores para devolver los productos usados. Masoudipour et al. [7] estudiaron las tasas de incentivos basadas en políticas de segmentación calculadas a partir de la calidad del artículo devuelto. Bhattacharya et al. [38] consideraron una red CSCC donde los artículos devueltos tenían diferentes niveles de calidad y las tasas de devolución se basaban en el incentivo ofrecido a los retornantes en cada etapa. Modak et al. [39] examinaron las estrategias de recolección en una red CSCC donde la tasa de retorno de los productos dependía de la inversión del minorista en las actividades de recolección. Taleizadeh et al. [10] consideraron una política de incentivos en la que los clientes recibían un descuento para comprar nuevos productos al devolver productos usados y el descuento ofrecido dependía de la calidad del producto devuelto. Ruiz-Torres et al. [17] propusieron un modelo que ayuda a administrar el flujo de devoluciones al determinar los incentivos para ofrecer a los retornantes, al tiempo que determina un contrato de capacidad con el proveedor de nuevos componentes.

2.2. Selección y asignación de órdenes a los proveedores.- El problema de selección de proveedores y la asignación de órdenes entre los proveedores seleccionados es uno de alta complejidad [40-42]. Para garantizar el suministro de materiales a lo largo de la cadena de suministro, el proceso de selección involucra varios factores y criterios conflictivos como el precio 
y la calidad de los materiales suministrados y la confiabilidad de las entregas. Muchos estudios han examinado este problema sin considerar los riesgos de falla de los proveedores [40]. Sin embargo, los flujos de material pueden verse afectados por desastres naturales inesperados o provocados por el hombre, como terremotos, incendios, inundaciones, huracanes, averías de equipos, huelgas laborales, crisis económica, quiebras, sabotaje o ataques terroristas [42].

Múltiples investigaciones han considerado modelos donde existe la posibilidad de falla en la entrega de los proveedores. Berger et al. [43] fue el primer estudio que consideró la probabilidad de falla del proveedor en el problema de selección de proveedores y consideró los riesgos asociados con una red de proveedores, que incluyó eventos catastróficos que afectan a todos los proveedores, así como eventos únicos que afectaron solo a un solo proveedor. A su vez, Ruiz-Torres y Mahmoodi [44] desarrollaron una extensión de este trabajo al proponer un modelo de decisión para optimizar la asignación de la demanda en un conjunto de proveedores al considerar tres factores de costo clave: las pérdidas esperadas debido a la falta de entrega del proveedor, los costos de compra y el costo de mantener un conjunto de proveedores. Moritz y Pibernik [45] expandieron en el modelo de Ruiz-Torres y Mahmoodi [44] asumiendo descuentos en los costos de los proveedores basándose en el volumen de la orden asignada a cada proveedor.

Por su parte, Sarkar y Mohapatra [46] y Meena et al. [47] abordaron el problema de determinar el número de proveedores bajo riesgos de falla del proveedor debido a eventos extremos y eventos únicos. Sawik [48] investigó la cartera de suministros óptima en un entorno de fabricación por pedido basado en el precio y la calidad de las piezas compradas, así como la confiabilidad de la entrega. Meena y Sarmah [49] desarrollaron un modelo de programación no lineal para la asignación de pedidos entre múltiples proveedores bajo riesgos de interrupción del suministro para minimizar el costo total considerando diferentes capacidades, probabilidades de fallas y descuentos por cantidad para cada proveedor. Sawik [42] propuso un modelo para la selección de una cartera de suministro resistente en una cadena de suministro con riesgos de interrupción. Ruiz-Torres et al. [50] analizaron un modelo en el que los artículos obtenidos se utilizan en múltiples instalaciones, se pueden comprar a múltiples proveedores con diferentes características de costo y confiabilidad, y los proveedores tenían flexibilidad de producción que les permitía entregar una cantidad de contingencia en caso de que otros proveedores fallaran.

Más adelante, Torabi et al. [51] desarrollaron un modelo de programación estocástica de dos etapas y dos objetivos para construir bases de suministro resistentes considerando las incertidumbres de las interrupciones importantes, así como los riesgos operativos. Kamalahmadi y Mellat-Parast [52] utilizaron un modelo para desarrollar estrategias de contingencia en caso de ocurrir eventos disruptivos, el modelo integra la selección de proveedores y localización de la demanda con selección de cadenas de transportación y desarrollo de planes de contingencia para mitigar y minimizar los costos de la red. Meena y Sarmah [53] desarrollaron un modelo analítico y un procedimiento de solución para determinar el número óptimo de proveedores y la asignación óptima de la demanda entre los proveedores seleccionados bajo los riesgos de falla del proveedor y consideraron diferentes probabilidades de falla, capacidad y descuento de precio basado en la cantidad para cada proveedor.

Más recientemente, Sawik [54] propone un modelo que selecciona un proveedor y determina cuándo entregar las piezas específicas del producto requeridas para cada pedido del cliente para cumplir con la fecha de vencimiento solicitada por el cliente. Esmaeili-Najafabadi et al. [55] desarrollaron un modelo de programación no lineal y consideran las políticas de protección e inventario de emergencia para optimizar la selección de proveedores y la asignación de pedidos con el fin de reducir los efectos de interrupción. Lücker et al. [56] determinaron los niveles óptimos de inventario y las tasas de producción de capacidad de reserva para una empresa que está expuesta

Memoria Investigaciones en Ingeniería, núm. 19 (2020). pp 2-24 
al riesgo de interrupción de la cadena de suministro; caracterizando cuatro estrategias principales de mitigación de riesgos: estrategia de inventario, estrategia de capacidad de reserva, estrategia mixta y aceptación pasiva. Finalmente, Hosseini et al. [57] calcularon la probabilidad de un escenario de interrupción de los proveedores utilizando un modelo gráfico probabilístico, y luego propusieron un modelo estocástico para apoyar la toma de decisiones en términos de cómo y cuándo usar estrategias proactivas y reactivas en selección de proveedores y asignación de pedidos.

2.3. Contribución a la literatura.- Este estudio combina elementos de las áreas de conocimiento descritas en las subsecciones anteriores. En particular el modelo que se propone considera una red CSCC donde se ofrecen incentivos como en Bhattacharya et al. [38], y estos incentivos directamente afectan la cantidad de los retornos. Como en Aras y Aksen [34] y Aras et al. [35] elementos de la decisión son los integrantes de la red, en este caso qué fuentes de retorno serán utilizadas. El modelo propuesto también considera decisiones asociadas con la cantidad a reservar de un proveedor de componentes nuevos cuando existen riesgos de no cumplir con la entrega como en Meena et al. [47] y Sawik [54].

El modelo propuesto en Ruiz-Torres et al. [17] es el único en la literatura que considera de manera integrada estas áreas; las decisiones asociadas con los incentivos que ofrecer a las fuentes de retorno y decisiones asociadas con el proveedor de nuevos componentes. El modelo propuesto en este estudio expande el trabajo de Ruiz-Torres et al. [17] al incluir decisiones asociadas con qué fuentes de retorno deben de ser utilizadas y al tener descuentos por la cantidad de capacidad reservada. Este trabajo de investigación es una contribución significativa a la literatura al proveer un modelo integrado de incentivos, diseño de red de retornos, y selección de capacidad de componentes nuevos.

3. Modelo propuesto.- En esta sección se describe el modelo propuesto y se presenta un ejemplo para demonstrar su funcionamiento. Este modelo considera la situación donde una empresa de manufactura requiere $d$ unidades de un componente cada ciclo de producción para satisfacer las necesidades de sus clientes. Este componente se puede obtener como un artículo nuevo o usado que va a ser remanufacturado internamente. Los componentes usados se reciben en una condición similar de desgaste y deterioro. Existen varias fuentes de retorno para el componente usado y cada fuente está relacionada con la ubicación donde se recogen los componentes. Los componentes usados se remanufacturan a una condición "nueva". Se utiliza un solo proveedor de componentes nuevos.

3.1. Fuentes de retorno.- El conjunto de fuentes de retorno es representado por $F$ y una decisión del modelo es cuales fuentes de retorno serán utilizadas. El conjunto de niveles de incentivos es representado por $I$. La cantidad de unidades retornadas por cada fuente es incierta y está relacionada con el incentivo que se ofrece. El conjunto de los niveles de cantidad de retorno es representado por $Z$. La variable $q_{z, i, f}$ es la cantidad de unidades retornadas en el nivel de retorno $Z$ cuando el nivel de incentivo $i$ se le ofrece a la fuente $f$.

El problema se modela como un árbol de decisión basándose en los niveles de retorno. El árbol incluye todas las fuentes de retorno ya que en el caso de que una o más fuentes no sean utilizadas, los incentivos y cantidades asociadas se convierten en 0. Cada combinación de los niveles de retorno es un posible escenario (estado de la naturaleza). Se define la variable $E$ como el conjunto de todos los posibles escenarios.

El modelo considera dos decisiones que la empresa va a tomar en relación con las fuentes de retorno: cuáles fuentes de retorno utilizar/operar y el incentivo que va a ofrecer en cada fuente. La 
variable $u_{f}$ representa una decisión binaria igual a 1 si la fuente de retorno $f$ será utilizada y es igual a 0 en caso contrario. La variable $a_{i, f}$ representa una decisión binaria igual a 1 si se ofrece el nivel de incentivo $i$ a la fuente de retorno $f$ y es igual a 0 en caso contrario. Las variables $u_{f}$ y $a_{i, f}$ son elementos de decisión de este modelo y solo se puede seleccionar un nivel de incentivo por fuente de retorno que esté operando, por lo tanto, es necesaria la siguiente restricción $\sum_{i \in I} a_{i, f}=u_{f} \forall f \in F$. La variable $r_{e}$ representa la cantidad total de retornos para el escenario $e$. La determinación de $r_{e}$ se basa en las variables de decisión mencionadas: $r_{e}=\sum_{f \in F, i \in I} a_{i, f} \times$ $q_{i e, i, f}$.

Para demostrar la implementación del modelo se asume una empresa con una demanda por ciclo de 500 unidades $(d=500)$, con 3 posibles fuentes de retorno $(F=\{f 1, f 2, f 3\})$, que existen tres niveles de incentivos $(I=\{$ Alto, Medio,Bajo $\})$ y que existen dos niveles de retorno $(Z=$ $\{$ Mucho,Poco $\}$ ). El número de escenarios es $|F|^{|I|}$ y la Figura I presenta todos los posibles escenarios basándose en los tres niveles de incentivos, por lo tanto, $E=$ $\{($ Mucho, Mucho, Mucho), (Mucho, Mucho, Poco $), \ldots,($ Poco, Poco, Poco $)\} . \quad$ Se define ze como los componentes de nivel de retorno para un escenario, por lo que $z \dot{1}=($ Mucho, Mucho, Mucho $), \dot{2}=($ Mucho, Mucho, Poco $), \dot{z}=($ Mucho, Poco, Mucho $), \ldots$.. La Tabla I provee la información de las cantidades por fuente, por incentivo, y por nivel de retorno. Los valores utilizados se desarrollaron tomando como base datos de una empresa que remanufacura copiadoras.

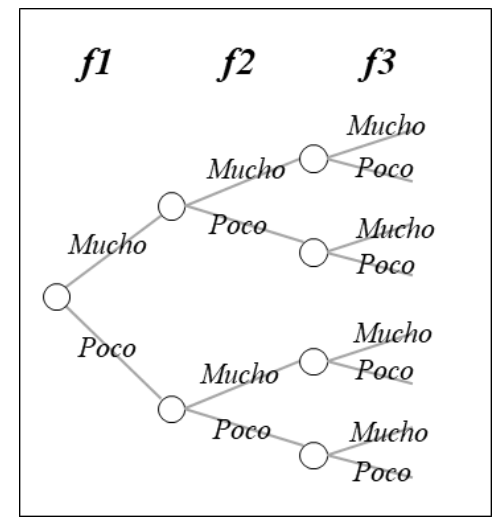

Figura I.- Árbol de decisión con escenarios posibles.

\begin{tabular}{|c|c|c|c|c|}
\hline \multicolumn{2}{|c|}{} & \multicolumn{3}{|c|}{ Incentivo (i) } \\
\hline Fuente (f) & Nivel de retorno (z) & Alto & Medio & Bajo \\
\hline \multirow{2}{*}{ f1 } & Mucho & 95 & 80 & 70 \\
\cline { 2 - 5 } & Poco & 72 & 62 & 50 \\
\hline \multirow{2}{*}{ f2 } & Mucho & 189 & 166 & 153 \\
\cline { 2 - 5 } & Poco & 126 & 112 & 90 \\
\hline \multirow{2}{*}{ 33 } & Mucho & 195 & 165 & 150 \\
\cline { 2 - 5 } & Poco & 125 & 115 & 100 \\
\hline
\end{tabular}

Tabla I.- Devolución prevista por fuente, nivel de retorno, y nivel de incentivo $\left(q_{z, i, f}\right)$.

De manera de demonstrar el modelo, se asume que la decisión de la empresa es de usar las tres 
fuentes de retorno $\left(u_{f 1}=u_{f 2}=u_{f 3}=1\right)$ y ofrecer un incentivo Alto para $f 1$, un incentivo Bajo para $f 2$ y un incentivo Medio para $f 3 \quad\left(a_{\text {Alto } f 1}=1, a_{B a j o, f 2}=1, a_{\text {Medio }, f 3}=\right.$ 1 , todos los otros $a_{i, f}=0$ ). En la Tabla II se presenta la cantidad de unidades por fuente y el $r_{e}$ para cada escenario en $E$. El máximo de unidades retornado bajo ese conjunto de decisiones es 413, mientras que el mínimo es 277.

\begin{tabular}{|c|c|c|c|c|c|}
\hline \multicolumn{2}{|c|}{} & \multicolumn{3}{|c|}{ Retornos por fuente } & \\
\hline$e$ & ze & $f 1$ & $f 2$ & $f 3$ & $r_{e}$ \\
\hline e1 & Mucho, Mucho, Mucho & 95 & 153 & 165 & 413 \\
\hline e2 & Mucho, Mucho, Poco & 95 & 153 & 115 & 363 \\
\hline e3 & Mucho, Poco, Mucho & 95 & 90 & 165 & 350 \\
\hline e4 & Mucho, Poco, Poco & 95 & 90 & 115 & 300 \\
\hline e5 & Poco, Mucho, Mucho & 72 & 153 & 165 & 390 \\
\hline e6 & Poco, Mucho, Poco & 72 & 153 & 115 & 340 \\
\hline e7 & Poco, Poco, Mucho & 72 & 90 & 165 & 327 \\
\hline e8 & Poco, Poco, Poco & 72 & 90 & 115 & 277 \\
\hline
\end{tabular}

Tabla II.- Cantidades de devolución prevista por nivel de retorno, nivel de incentivo y fuente para el caso 1 de ejemplo $\left(q_{z, i, f}\right)$.

3.2. Proveedor de unidades nuevas.- El modelo considera una decisión asociada con el proveedor de unidades nuevas: la capacidad reservada. El proveedor ofrece descuentos relacionados a la capacidad reservada, mientras mayor la capacidad, mayor el descuento. La variable $n$ representa la capacidad reservada, la variable $T$ representa el conjunto de posibles cantidades que pueden ser reservadas incluyendo 0 , y la variable $n_{t}$ define la capacidad reservada en el nivel $t$. La variable $h_{t}$ representa una decisión binaria igual a 1 si se selecciona el nivel $t$ como la capacidad reservada y es igual a 0 en caso contrario. Solo una cantidad puede ser reservada por lo cual es necesaria la siguiente restricción: $\sum_{t \in T} h_{t}=1$ y la variable $n$ se determina de esta manera $n=\sum_{t \in T} n_{t} \times h_{t}$.

La capacidad reservada no se utiliza bajo cada posible escenario ya que es una función del total de unidades retornadas $r_{e}$. La variable $k_{e}$ representa el número de componentes nuevos que son ordenados a proveedor en el escenario $e$. La cantidad $k_{e}$ se define por $\boldsymbol{\operatorname { m i n }}\left[d-r_{e}, \quad n\right]$; las unidades requeridas del proveedor deben de ser menor o igual que la demanda no satisfecha por los retornos, o la capacidad fija reservada. Se asume que la demanda es siempre mayor que los retornos.

El modelo considera la incertidumbre asociada con la falla de entrega del proveedor. La variable $w$ representa la probabilidad de que no se reciban las unidades requeridas del proveedor (causas internas o externas). Cuando el proveedor falla, la demanda no satisfecha se define por $d-r_{e}$ (la demanda menos el total de los retornos), mientras que la variable $m_{e}$ representa la demanda no satisfecha cuando el proveedor entrega; $m_{e}=d-r_{e}-k_{e}$ (la demanda menos el total de los retornos menos el total de componentes nuevos recibidos del proveedor).

Para el ejemplo se asume que el proveedor permite reservar capacidad en "lotes" de 100 unidades: $T=\{t 1=0, t 2=100, t 3=200, \ldots, t 6=500\}$. Se asume un nivel máximo de $t 6=d=500$ ya que nunca haría falta ordenar más que la demanda. Se asume como ejemplo que la empresa se decide por $n=200\left(h_{t 3}=1\right.$, todos los otros $\left.h_{t}=0\right)$. La Tabla III presenta por escenario el 
total de unidades retornadas $\left(r_{e}\right)$, la demanda no satisfecha cuando el proveedor no entrega $(d-$ $\left.r_{e}\right)$, la cantidad requerida y entregada por al proveedor $\left(k_{e}\right)$, y la demanda no satisfecha cuando no se reciben las unidades requeridas del proveedor $\left(m_{e}\right)$. En 7 de los escenarios cuando el proveedor entrega se cumple totalmente con la demanda; en el escenario $e 8$ hay una demanda no satisfecha aun cuando se reciben las unidades requeridas al proveedor.

\begin{tabular}{|c|c|c|c|c|c|}
\hline$e$ & $i e$ & $r_{e}$ & $d-r_{e}$ & $k_{e}$ & $m_{e}$ \\
\hline e1 & Mucho, Mucho, Mucho & 413 & 87 & 87 & 0 \\
\hline e2 & Mucho, Mucho, Poco & 363 & 137 & 137 & 0 \\
\hline e3 & Mucho, Poco, Mucho & 350 & 150 & 150 & 0 \\
\hline e4 & Mucho, Poco, Poco & 300 & 200 & 200 & 0 \\
\hline e5 & Poco, Mucho, Mucho & 390 & 110 & 110 & 0 \\
\hline e6 & Poco, Mucho, Poco & 340 & 160 & 160 & 0 \\
\hline e7 & Poco, Poco, Mucho & 327 & 173 & 173 & 0 \\
\hline e8 & Poco, Poco, Poco & 277 & 223 & 200 & 23 \\
\hline
\end{tabular}

Tabla III.- Total por escenario de las unidades retornadas $\left(r_{e}\right)$, demanda no satisfecha cuando no se reciben las unidades requeridas al proveedor $\left(d-r_{e}\right)$, cantidad requerida y entregada por el proveedor

$\left(k_{e}\right)$, y demanda no satisfecha cuando se reciben las unidades requeridas al proveedor $\left(m_{e}\right)$.

3.3. Elementos de Costo.- El modelo considera tres costos asociados con las fuentes de retorno: a) costo fijo asociado con operar cada fuente de retorno; b) costo por unidad asociado con transportar y remanufacturar las unidades provenientes de cada fuente y c) costo de los incentivos que se pagan por unidad retornada. En relación con el proveedor se consideran dos costos: a) costo de reservar capacidad y b) costo por unidad requerida y entregada por el proveedor. Finalmente, el modelo considera el costo asociado con la pérdida de órdenes y clientes futuros basándose en la demanda no satisfecha. Se definen los siguientes parámetros de costo:
$c_{f}^{\text {oper }}$
$c_{f}^{\text {tr-rem }}$
Costo fijo para la operación de la fuente de retorno $f$.
Costo de transportación/remanufactura por unidad proveniente de la fuente de retorno $f$.
$c_{i, f}^{i n c e} \quad$ Costo de incentivo por unidad en el nivel $i$ para la fuente de retorno $f$.
$c_{t}^{\text {rese }} \quad$ Costo por unidad de capacidad reservada para el nivel $t$.
$c^{\text {entr }} \quad$ Costo por unidad requerida y entregada por el proveedor.
$c^{\text {perd }} \quad$ Costo por unidad de la demanda no satisfecha.

Los costos tomando las decisiones de la empresa se dividen en costos fijos y costos por escenario (variables). Hay dos costos fijos: costo de operar los centros de retorno y el costo de reservar capacidad.
$c t^{\text {oper }}$
Costo total de operación de las fuentes de retorno.
$c t^{\text {rese }}$
Costo total de reservar capacidad.

Donde:

$$
\begin{aligned}
& c t^{\text {oper }}=\sum_{f \in F} u_{f} \times c_{f}^{\text {oper }}+\sum_{t \in T} h_{t} \times c_{t}^{\text {rese }} \\
& c t^{\text {rese }}=\sum_{t \in T} h_{t} \times n \times c_{t}^{\text {rese }}
\end{aligned}
$$

Hay cuatro componentes de los costos por escenario (variables): costo de transportar/ 
remanufacturar, costos de incentivos, costo de las unidades recibidas del proveedor cuando entrega, y costos de pérdida por la demanda no satisfecha. Las ecuaciones de los costos por escenario $e$ son:

$\begin{array}{ll}c t_{e}^{\text {tr-rem }} & \text { Costo total de transportar/ remanufacturar. } \\ c t_{e}^{\text {ince }} & \text { Costo total de los incentivos. } \\ c t^{\text {entr }} & \text { Costo total por la unidades requeridas y entregadas por el proveedor. } \\ c t^{\text {perd }} & \text { Costo total por demanda no satisfecha. }\end{array}$

Donde:

$$
\begin{aligned}
& c t_{e}^{\text {tr-rem }}=\sum_{i \in I, f \in F} a_{i, f} \times q_{i e, i, f} \times c_{f}^{\text {tr-rem }} \\
& c t_{e}^{\text {ince }}=\sum_{i \in I, f \in F} a_{i, f} \times q_{\dot{z} e, i, f} \times c_{i, f}^{\text {ince }} \\
& c t^{\text {entr }}=(1-w) \times k_{e} \times c^{\text {entr }} \\
& c t^{\text {perd }}=\left((1-w) \times m_{e}+w \times\left(d-r_{e}\right)\right) \times c^{\text {perd }} .
\end{aligned}
$$

Por lo tanto, el costo variable para el escenario $e$ es $c t_{e}^{\text {tr-rem }}+c t_{e}^{\text {ince }}+c t^{e n t r}+c t^{\text {perd }}$. El total de costos variables es basado en el valor esperado sobre todos los posibles escenarios. Cada escenario del modelo tiene una probabilidad en función de las decisiones de incentivo de la empresa. La variable $p_{z, i, f}$ representa la probabilidad del nivel de retorno $z$ al ofrecer el nivel de incentivo $i$ a la fuente $f$. Es importante notar que la suma de las probabilidades de los niveles de retorno asociadas con cada fuente y nivel de incentivo suman igual a 1: $\sum_{z \in Z} p_{z, i, f}=1 \forall i \in$ $I, \forall f \in F$. La variable $p_{e}$ representa la probabilidad del escenario $e$, donde $p_{e}=$ $\prod_{f \in F}\left(\left(1-u_{f}\right) /|Z|+\sum_{i \in I}\left(a_{i, f} \times p_{z e, i, f}\right)\right)$. El componente $\left(1-u_{f}\right) /|Z|$ se utiliza para modelar la probabilidad de fuentes que no están en operación y de donde no se recibirán unidades sin importar el escenario.

La variable $\Theta$ representa el costo total del sistema; $\Theta=c t^{\text {oper }}+c t^{\text {rese }}+\sum_{e \in E}\left(\left(c t_{e}^{\text {tr-rem }}+\right.\right.$ $\left.c t_{e}^{\text {ince }}+c t^{\text {entr }}+c t^{\text {perd }}\right) \times p_{e}$ ). El objetivo del modelo es minimizar $\Theta$.

Para el ejemplo se asume que la probabilidad de que no se reciban las unidades del proveedor es $5 \%(w=5 \%)$, el costo por unidad de la demanda no satisfecha es $\$ 90\left(c^{\text {perd }}=\$ 90\right)$ y los costos por fuente de retorno están presentados en la Tabla IV. El costo de reservar capacidad en el nivel $t 2$ es $\$ 32\left(c_{t 2}^{r e s e}=\$ 32\right)$ y disminuye $\$ 1$ por nivel, por lo tanto, en $t 6$ es $\$ 28\left(c_{t 6}^{r e s e}=\$ 28\right)$. El nivel $t 1$ es equivalente a una reservación de capacidad de 0 , por lo que $c_{t 1}^{\text {rese }}$ es 0 . El costo por unidad requerida y entregada es $\$ 8\left(c^{\text {entr }}=\$ 8\right)$.

\begin{tabular}{|c|c|c|c|c|c|}
\hline Fuente $(f)$ & $c_{f}^{\text {oper }}$ & $c_{f}^{\text {tr-rem }}$ & $c_{\text {Alto,f }}^{\text {ince }}$ & $c_{\text {Medio,f }}^{\text {ince }}$ & $c_{\text {Bajo,f }}^{\text {ince }}$ \\
\hline$f 1$ & $\$ 1,860$ & $\$ 4$ & $\$ 10.0$ & $\$ 5.0$ & $\$ 1.5$ \\
\hline$f 2$ & $\$ 2,260$ & $\$ 5$ & $\$ 6.0$ & $\$ 3.0$ & $\$ 0.75$ \\
\hline$f 3$ & $\$ 2,790$ & $\$ 7$ & $\$ 9.0$ & $\$ 4.5$ & $\$ 1.25$ \\
\hline
\end{tabular}

Tabla IV.- Parámetros de costo relacionados a las fuentes de retorno.

Basado en las decisiones de la empresa de operar todos los centros de retorno $\left(u_{f 1}=u_{f 2}=u_{f 3}=\right.$ 1) y de reservar capacidad al nivel $t 3\left(h_{t 3}=1\right.$, todos los otros $\left.h_{t}=0\right)$, los costos fijos son los siguientes:ct ${ }^{\text {oper }}=\$ 1,860+\$ 2,260+\$ 2,790=\$ 6,910$ y $c t^{\text {rese }}=200 \times \$ 31=\$ 6,200$. La Tabla $\mathrm{V}$ presenta los cuatro costos variables y su suma por escenario. 
Por ejemplo, en $e 8$ se reciben 72,90, y 115 de $f 1, f 2$, y $f 3$ respectivamente $\left(q_{\text {Poco,Alto,f } 1}=\right.$ $\left.72, q_{\text {Poco }, \text { Bajo,f } 2}=90, q_{\text {Poco }, \text { Medio }, f 3}=115\right)$. Por lo tanto, $c t_{e 8}^{\text {tr-rem }}=72 \times \$ 4+90 \times \$ 5+$ $115 \times \$ 7=\$ 1,543$ y $c t_{e 8}^{i n c e}=72 \times \$ 10+90 \times \$ 0.75+115 \times \$ 4.5=\$ 1,305$. En $e 8$ se requieren 200 unidades del proveedor $\left(k_{e 8}=200\right)$, por lo tanto, $c t^{e n t r}=95 \% \times 200 \times \$ 8=$ $\$ 1,520$. En $e 8$ cuando se reciben las unidades del proveedor, la demanda no satisfecha es 23 unidades $\left(m_{e 8}=23\right)$ y cuando no se reciben la demanda no satisfecha es 223 unidades $\left(d-r_{e 8}=\right.$ 223), por lo tanto $c t^{\text {perd }}=(95 \% \times 23+5 \% \times 223) \times \$ 90=\$ 2,970$.

\begin{tabular}{|c|c|c|c|c|c|c|}
\hline$e$ & $i e$ & $c t_{e}^{\text {tr-rem }}$ & $c t_{e}^{\text {ince }}$ & $c t^{\text {entr }}$ & $c t^{\text {perd }}$ & Suma \\
\hline e1 & Mucho, Mucho, Mucho & $\$ 2,300$ & $\$ 1,807$ & $\$ 661$ & $\$ 392$ & $\$ 5,160$ \\
\hline e2 & Mucho, Mucho, Poco & $\$ 1,950$ & $\$ 1,582$ & $\$ 1,041$ & $\$ 617$ & $\$ 5,190$ \\
\hline e3 & Mucho, Poco, Mucho & $\$ 1,985$ & $\$ 1,760$ & $\$ 1,140$ & $\$ 675$ & $\$ 5,560$ \\
\hline e4 & Mucho, Poco, Poco & $\$ 1,635$ & $\$ 1,535$ & $\$ 1,520$ & $\$ 900$ & $\$ 5,590$ \\
\hline e5 & Poco, Mucho, Mucho & $\$ 2,208$ & $\$ 1,577$ & $\$ 836$ & $\$ 495$ & $\$ 5,116$ \\
\hline e6 & Poco, Mucho, Poco & $\$ 1,858$ & $\$ 1,352$ & $\$ 1,216$ & $\$ 720$ & $\$ 5,146$ \\
\hline e7 & Poco, Poco, Mucho & $\$ 1,893$ & $\$ 1,530$ & $\$ 1,315$ & $\$ 779$ & $\$ 5,516$ \\
\hline e8 & Poco, Poco, Poco & $\$ 1,543$ & $\$ 1,305$ & $\$ 1,520$ & $\$ 2,970$ & $\$ 7,338$ \\
\hline
\end{tabular}

Tabla V.- Costos variables por escenario para el ejemplo.

La Tabla VI presenta las probabilidades por escenario para el nivel Poco, donde $p_{M u c h o, i, f}=1-$ $p_{\text {Poco,i,f }} \forall i \in I, \forall f \in F$ (recordando que las probabilidades para los niveles de retorno para cada fuente y nivel de incentivo suman igual a 1). Es importante notar que el modelo no condiciona la relación entre las probabilidades de entrega y los niveles de incentivos, pero es lógico asumir que el valor esperado de las unidades retornadas por nivel de incentivo y fuente $\left(\sum_{z \in Z} q_{z, i, f} \times p_{z, i, f}\right)$ aumentan a medida que los incentivos aumentan. Por ejemplo, en el caso de $f 2$, la probabilidad del nivel de retorno Mucho disminuye al aumentar los incentivos $\left(p_{M u c h o, B a j o, f 2}=65 \%\right.$, $\left.p_{\text {Mucho,Medio,f } 2}=55 \%, p_{\text {Mucho,Alto,f } 2}=45 \%\right)$, pero el valor esperado de unidades aumenta. El valor esperado de unidades para el nivel de incentivos Bajo es $130.9(65 \% \times 153+$ $35 \% \times 90)$, para el nivel de incentivos Medio es $141.7(55 \% \times 166+45 \% \times 112)$ y para 11 nivel de incentivo Alto es $154.4(45 \% \times 189+55 \% \times 126)$.

\begin{tabular}{|c|c|c|c|}
\hline & \multicolumn{3}{|c|}{ Incentivo (i) } \\
\hline Fuente $(f)$ & Alto & Medio & Bajo \\
\hline$f 1$ & $35 \%$ & $40 \%$ & $60 \%$ \\
\hline$f 2$ & $55 \%$ & $45 \%$ & $35 \%$ \\
\hline$f 3$ & $65 \%$ & $60 \%$ & $90 \%$ \\
\hline
\end{tabular}

Tabla VI.- Probabilidad del nivel de retorno Poco por nivel de incentivo y fuente $\left(p_{P o c o, i, f}\right)$.

La Tabla VII presenta las probabilidades que aplican por escenario dadas las decisiones de la empresa $\left(a_{\text {Alto }, f 1}=1, a_{\text {Bajo,f } 2}=1, a_{\text {Medio, } f 3}=1\right.$, todos los otros $\left.a_{i, f}=0\right)$.

Para determinar el costo total se suman los costos fijos al valor esperado de los costos por escenario: $\Theta=\$ 6,910+\$ 6,200+(\$ 5,160 \times 16.9 \%+\$ 5,190 \times 25.4 \%+\cdots+\$ 7,338 \times 7.4 \%)=$ $\$ 18,544$. 


\begin{tabular}{|c|c|c|c|c|c|}
\hline$e$ & $i e$ & $p_{z, \text { Alto,f } 1}$ & $p_{z, \text { Bajo,f } 2}$ & $p_{z, \text { Medio,f } 3}$ & $p_{e}$ \\
\hline e1 & Mucho, Mucho, Mucho & $65 \%$ & $65 \%$ & $40 \%$ & $16.9 \%$ \\
\hline e2 & Mucho, Mucho, Poco & $65 \%$ & $65 \%$ & $60 \%$ & $25.4 \%$ \\
\hline e3 & Mucho, Poco, Mucho & $65 \%$ & $35 \%$ & $40 \%$ & $9.1 \%$ \\
\hline e4 & Mucho, Poco, Poco & $65 \%$ & $35 \%$ & $60 \%$ & $13.7 \%$ \\
\hline e5 & Poco, Mucho, Mucho & $35 \%$ & $65 \%$ & $40 \%$ & $9.1 \%$ \\
\hline e6 & Poco, Mucho, Poco & $35 \%$ & $65 \%$ & $60 \%$ & $13.7 \%$ \\
\hline e7 & Poco, Poco, Mucho & $35 \%$ & $35 \%$ & $40 \%$ & $4.9 \%$ \\
\hline e8 & Poco, Poco, Poco & $35 \%$ & $35 \%$ & $60 \%$ & $7.4 \%$ \\
\hline
\end{tabular}

Tabla VII.- Probabilidad de cada escenario $\left(p_{e}\right)$.

Se presenta ahora un segundo ejemplo (segundo conjunto de decisiones): la empresa decide solo usar las fuentes $f 2$ y $f 3\left(u_{f 1}=0, u_{f 2}=u_{f 3}=1\right)$ y para ambas se les ofrece el nivel de incentivo Alto, $\quad\left(a_{\text {Alto }, f 2}=a_{\text {Alto,f3 }}=1\right.$, todos los otros $\left.a_{i, f}=0\right)$. Además, la empresa continuará reservando 200 unidades $(n=200)$. Esto significa que los costos fijos son los siguientes: ct $^{\text {oper }}=$ $\$ 2,260+\$ 2,790=\$ 5,050$ y $c t^{\text {rese }}=200 \times \$ 31=\$ 6,200$.

La Tabla VIII presenta las cantidades retornadas en este segundo ejemplo donde el máximo número de unidades retornadas es 384 y el mínimo es 251 . Es importante notar que dado que $f 1$ no opera en este caso, hay en el sentido "práctico" solo 4 escenarios. El escenario $e 1$ y el $e 5$ son iguales, el $e 2$ y el $e 6$ son iguales y así sucesivamente.

\begin{tabular}{|c|c|c|c|c|c|}
\hline \multicolumn{2}{|c|}{ ze } & \multicolumn{3}{|c|}{ Retornos por fuente } & \\
\hline$e$ & Mucho, Mucho, Mucho & 0 & 189 & 195 & 384 \\
\hline e1 & Mucho, Mucho, Poco & 0 & 189 & 125 & 314 \\
\hline e2 & Mucho, Poco, Mucho & 0 & 126 & 195 & 321 \\
\hline e3 & Mucho, Poco, Poco & 0 & 126 & 125 & 251 \\
\hline e4 & Poco, Mucho, Mucho & 0 & 189 & 195 & 384 \\
\hline e5 & Poco, Mucho, Poco & 0 & 189 & 125 & 314 \\
\hline e6 & Poco, Poco, Mucho & 0 & 126 & 195 & 321 \\
\hline e7 & Poco, Poco, Poco & 0 & 126 & 125 & 251 \\
\hline e8 &
\end{tabular}

Tabla VIII.- Cantidades de devolución previstas por nivel de retorno, nivel de incentivo y fuente para el segundo ejemplo $\left(q_{z, i, f}\right)$.

La Tabla IX presenta por escenario el total de unidades retornadas $\left(r_{e}\right)$, la demanda no satisfecha cuando no se reciben las unidades requeridas del proveedor $\left(d-r_{e}\right)$, la cantidad requerida y entregada por al proveedor $\left(k_{e}\right)$, y la demanda no satisfecha cuando se reciben las unidades requeridas del proveedor $\left(m_{e}\right)$. En los escenarios $e 4$ y $e 8$ cuando se reciben las unidades requeridas del proveedor no se cumple totalmente con la demanda. La Tabla X presenta los cuatro costos variables y su suma por escenario. Al comparar los valores con los de la Tabla V (primer conjunto de decisiones de la empresa), estos costos son mayores para cada escenario. 


\begin{tabular}{|c|c|c|c|c|c|}
\hline$e$ & $\dot{z} e$ & $r_{e}$ & $d-r_{e}$ & $k_{e}$ & $m_{e}$ \\
\hline e1 & Mucho, Mucho, Mucho & 384 & 116 & 116 & 0 \\
\hline e2 & Mucho, Mucho, Poco & 314 & 186 & 186 & 0 \\
\hline e3 & Mucho, Poco, Mucho & 321 & 179 & 179 & 0 \\
\hline e4 & Mucho, Poco, Poco & 251 & 249 & 200 & 49 \\
\hline e5 & Poco, Mucho, Mucho & 384 & 116 & 116 & 0 \\
\hline e6 & Poco, Mucho, Poco & 314 & 186 & 186 & 0 \\
\hline e7 & Poco, Poco, Mucho & 321 & 179 & 179 & 0 \\
\hline e8 & Poco, Poco, Poco & 251 & 249 & 200 & 49 \\
\hline
\end{tabular}

Tabla IX.- Total de unidades retornadas $\left(r_{e}\right)$, demanda no satisfecha cuando el proveedor no entrega $(d-$ $\left.r_{e}\right)$, cantidad requerida y entregada por al proveedor $\left(k_{e}\right)$, y demanda no satisfecha cuando el proveedor entrega $\left(m_{e}\right)$ para el segundo ejemplo.

\begin{tabular}{|c|c|c|c|c|c|c|}
\hline$e$ & $i e$ & $c t_{e}^{\text {tr-rem }}$ & $c t_{e}^{\text {ince }}$ & $c t^{\text {entr }}$ & $c t^{\text {perd }}$ & Suma \\
\hline e1 & Mucho, Mucho, Mucho & $\$ 2,310$ & $\$ 2,889$ & $\$ 882$ & $\$ 522$ & $\$ 6,603$ \\
\hline e2 & Mucho, Mucho, Poco & $\$ 1,820$ & $\$ 2,259$ & $\$ 1,414$ & $\$ 837$ & $\$ 6,330$ \\
\hline e3 & Mucho, Poco, Mucho & $\$ 1,995$ & $\$ 2,511$ & $\$ 1,360$ & $\$ 806$ & $\$ 6,672$ \\
\hline e4 & Mucho, Poco, Poco & $\$ 1,505$ & $\$ 1,881$ & $\$ 1,520$ & $\$ 5,310$ & $\$ 10,216$ \\
\hline e5 & Poco, Mucho, Mucho & $\$ 2,310$ & $\$ 2,889$ & $\$ 882$ & $\$ 522$ & $\$ 6,603$ \\
\hline e6 & Poco, Mucho, Poco & $\$ 1,820$ & $\$ 2,259$ & $\$ 1,414$ & $\$ 837$ & $\$ 6,330$ \\
\hline e7 & Poco, Poco, Mucho & $\$ 1,995$ & $\$ 2,511$ & $\$ 1,360$ & $\$ 806$ & $\$ 6,672$ \\
\hline e8 & Poco, Poco, Poco & $\$ 1,505$ & $\$ 1,881$ & $\$ 1,520$ & $\$ 5,310$ & $\$ 10,216$ \\
\hline
\end{tabular}

Tabla X.- Costos variables por escenario para el segundo ejemplo.

En la Tabla XI se presentan las probabilidades por escenario. Dado a que no hay incentivos en la fuente $f 1$ al no estar en operacion $\left(u_{f 1}=0\right)$, se presenta el componente $\left(1-u_{f}\right) /|Z|$. En este segundo ejemplo el costo total es: $\Theta=\$ 5,050+\$ 6,200+(\$ 6,603 \times 7.9 \%+\$ 6,330 \times$ $14.6 \%+\cdots+\$ 10,216 \times 17.9 \%)=\$ 19,078$. Por lo tanto, este segundo conjunto de decisiones, aunque redujo los costos fijos, incrementa los costos totales.

\begin{tabular}{|c|c|c|c|c|c|}
\hline$e$ & $z e$ & $\left(1-u_{f 1}\right) /|Z|$ & $p_{z, \text { Alto,f } 2}$ & $p_{z, \text { Alto,f3 }}$ & $p_{e}$ \\
\hline e1 & Mucho, Mucho, Mucho & $50 \%$ & $45 \%$ & $35 \%$ & $7.9 \%$ \\
\hline e2 & Mucho, Mucho, Poco & $50 \%$ & $45 \%$ & $65 \%$ & $14.6 \%$ \\
\hline e3 & Mucho, Poco, Mucho & $50 \%$ & $55 \%$ & $35 \%$ & $9.6 \%$ \\
\hline e4 & Mucho, Poco, Poco & $50 \%$ & $55 \%$ & $65 \%$ & $17.9 \%$ \\
\hline e5 & Poco, Mucho, Mucho & $50 \%$ & $45 \%$ & $35 \%$ & $7.9 \%$ \\
\hline e6 & Poco, Mucho, Poco & $50 \%$ & $45 \%$ & $65 \%$ & $14.6 \%$ \\
\hline e7 & Poco, Poco, Mucho & $50 \%$ & $55 \%$ & $35 \%$ & $9.6 \%$ \\
\hline e8 & Poco, Poco, Poco & $50 \%$ & $55 \%$ & $65 \%$ & $17.9 \%$ \\
\hline
\end{tabular}

Tabla XI.- Probabilidad de cada escenario, segundo ejemplo $\left(p_{e}\right)$.

Memoria Investigaciones en Ingeniería, núm. 19 (2020). pp 2-24

https://doi.org/10.36561/ING.19.2

SSN 2301-1092 • ISSN (en línea) 2301-1106 
3.4. Glosario de variables y parámetros.- El modelo tiene tres variables de decisión, todas de tipo binarias:
$u_{f}$
La variable es igual a 1 si la fuente de retorno $f$ será utilizada y es igual a 0 en caso contrario.
$h_{t} \quad$ La variable es igual a 1 si se selecciona el nivel $t$ como la capacidad reservada y es igual a 0 en caso contrario.
$a_{i, f} \quad$ La variable es igual a 1 si se ofrece el nivel de incentivo $i$ a la fuente de retorno $f$ y es igual a 0 en caso contrario.

Parámetros del modelo:
$d \quad$ Unidades requeridas del componente cada ciclo de producción
F $\quad$ Conjunto de fuentes de retorno
I $\quad$ Conjunto de niveles de incentivos.
$Z \quad$ Conjunto de niveles de cantidad de retorno.
$q_{z, i, f} \quad$ Cantidad de unidades retornadas en el nivel de retorno $z$ cuando el nivel de incentivo $i$ se le ofrece a la fuente $f$.
$r_{e} \quad$ Cantidad total de retornos para el escenario $e$.
$n \quad$ Capacidad reservada.
$T \quad$ Conjunto de posibles cantidades que pueden ser reservadas.
$n_{t} \quad$ Capacidad reservada en el nivel $t$.

3.5. Formulación del Modelo.- La función objetivo es $\operatorname{MIN}(\Theta)$ sujeto a las siguientes restricciones:

$$
\begin{array}{ll}
\Theta=c t^{\text {oper }}+c t^{\text {rese }}+\sum_{e \in E}\left(\left(c t_{e}^{t r-r e m}+c t_{e}^{\text {ince }}+c t^{\text {entr }}+c t^{\text {perd }}\right) \times p_{e}\right) \\
\sum_{i \in I} a_{i, f}=u_{f} & \forall f \in F \\
\sum_{f \in F, i \in I} a_{i, f} \times q_{i e, i, f}=r_{e} & \forall e \in E \\
\sum_{t \in T} h_{t}=1 & \\
\sum_{t \in T} n_{t} \times h_{t}=n & \\
k_{e} \leq n & \forall e \in E \\
k_{e}+m_{e}+r_{e}=d & \forall e \in E \\
m_{e}, k_{e} \geq 0 & \forall f \in F \\
u_{f} \in\{0,1\} & \forall t \in T \\
h_{t} \in\{0,1\} & \forall i \in I, \forall f \in F \\
a_{i, f} \in\{0,1\} &
\end{array}
$$

La restricción (1) garantiza que solo se utiliza un incentivo por fuente de retorno en operación y la restricción (2) establece el número de retornos por escenario. La restricción (3) garantiza que solo se reserva un nivel de capacidad del proveedor y la restricción (4) establece la cantidad reservada. La restricción (5) establece que lo que será requerido del proveedor será menor o igual a la cantidad reservada. La restricción (6) establece que la suma de las unidades requeridas, la demanda no satisfecha y el total de unidades retornadas son iguales a la demanda. La restricción (7) establece la no negatividad mientras que las restricciones (8), (9) y (10) establecen las variables binarias.

4. Análisis del modelo.- En esta sección se realiza un estudio de sensibilidad usando como base el ejemplo numérico descrito en la sección 3. Las cantidades de devolución previstas por fuente, nivel 
de retorno, y nivel de incentivo fueron presentadas en la Tabla I, los parámetros de costo relacionados a las fuentes de retorno fueron presentados en la Tabla IV, y las probabilidades por nivel de retorno, nivel de incentivo y fuente fueron presentados en la Tabla VI. La probabilidad de que no se reciban las unidades del proveedor es $5 \%(w=5 \%)$, el costo por unidad de la demanda no satisfecha es $\$ 90$ ( $\left.c^{\text {perd }}=\$ 90\right)$, y se reserva en "lotes" de 100 donde el nivel $t 1$ es equivalente a una reservación de capacidad de 0 , por lo que $c_{t 1}^{\text {rese }}$ es 0 y el costo de reservar capacidad en el nivel $t 2$ es $\$ 32\left(c_{t 2}^{\text {rese }}=\$ 32\right)$, disminuyendo $\$ 1$ por nivel. Finalmente, el costo por unidad requerida y entregada es $\$ 8$ ( $c^{\text {entr }}=\$ 8$ ). La solución óptima utilizando el modelo matemático es el uso de las tres fuentes de retorno $\left(u_{f 1}=u_{f 2}=u_{f 3}=1\right)$ y ofrecer un incentivo Medio para $f 1$ y $f 2$, y un incentivo Bajo para $f 3 \quad\left(a_{\text {Medio } f 1}=1, a_{\text {Medio }, f 2}=1, a_{\text {Bajo,f } 3}=\right.$ 1 , todos los otros $a_{i, f}=0$ ). La capacidad reservada fue de $n=200$. Estas decisiones resultaron en $\Theta=\$ 18,356$, lo cual, al ser la solución óptima, es un valor menor a los costos de las dos decisiones presentadas en la sección anterior. Las soluciones óptimas se determinaron utilizando MS Excel 365 con una rutina en VBA en una computadora personal con un RAM de $12 \mathrm{~GB}$ y un procesador de $2.7 \mathrm{GHz}$.

Las siguientes secciones analizan cómo cambian las decisiones del modelo al variar distintos parámetros del ejemplo.

4.1. Fuentes de Retorno.- Se analizan cambios a dos elementos de las fuentes de retorno:

a) los costos fijos de operación

b) la probabilidad de los niveles de retorno.

Se modifican los costos fijos de las fuentes de retorno por un factor $\alpha$, un incremento en un porcentaje de la base. El costo fijo de operación se determina por $c_{f 1}^{\text {oper }}=\$ 1,860 \times(1+$ $\alpha), c_{f 2}^{\text {oper }}=\$ 2,260 \times(1+\alpha), c_{f 3}^{\text {oper }}=\$ 2,790 \times(1+\alpha)$. El nivel $\alpha=0$ es el parámetro base y se evalúan los siguientes niveles de $\alpha: 10 \%, 20 \%, 30 \%$ y $40 \%$.

Se modifica la probabilidad del nivel Poco por un factor $\beta$ para todos los niveles de incentivos y fuentes. La probabilidad $p_{\text {Poco }, i, f}$ se basa entonces en la Tabla XII. El nivel $\beta=1$ es el parametro base y se evalúa el efecto de $\beta=0.8$ y $\beta=0.6$, lo que implica una reducción en la probabilidad de un nivel de retorno Poco de $20 \%$ y $40 \%$ respectivamentee.

\begin{tabular}{|c|c|c|c|}
\hline & \multicolumn{3}{|c|}{ Incentivo (i) } \\
\hline Fuente $(f)$ & Alto & Medio & Bajo \\
\hline$f 1$ & $35 \% \times \beta$ & $40 \% \times \beta$ & $60 \% \times \beta$ \\
\hline$f 2$ & $55 \% \times \beta$ & $45 \% \times \beta$ & $35 \% \times \beta$ \\
\hline$f 3$ & $65 \% \times \beta$ & $60 \% \times \beta$ & $90 \% \times \beta$ \\
\hline
\end{tabular}

Tabla XII.- Probabilidad del nivel de retorno Poco por nivel de incentivo y fuente $\left(p_{P o c o, i, f}\right)$.

La Tabla XIII presenta las decisiones para las 15 combinaciones de los niveles de prueba de $\alpha$ y $\beta$. Las decisiones indican el número de fuentes de retorno en operación, los niveles de incentivo por fuente (abreviado, $A=$ Alto, $M=$ Medio, $B=$ Bajo), y la capacidad reservada del proveedor.

Seis decisiones son óptimas dentro de los niveles de $\alpha$ y $\beta$ bajo análisis: $3(M / M / B), 200 ; 3(A /$ $A / M), 100 ; 2(M / M /-), 300 ; 2(-/ A / M), 200 ; 1(-/ M /-), 400 ; 0(-/-/-), 500$; donde la 
esquina superior izquierda es la solución base: $3(M / M / B), 200$. Como es de esperarse, cuando la probabilidad de retornos bajos disminuye $(\beta \downarrow)$, se ofrecen más incentivos y se reserva menos capacidad, mientras que, al aumentar los costos de operación de las fuentes de retorno, se reduce el número de fuentes de retorno en operación. En 9 de los 15 puntos experimentales es óptimo el usar 3 fuentes de retorno, mientras que en 2 de los 15 casos no se utiliza ninguna. Cuando la probabilidad de retornos está al nivel base $(\beta=1)$ y los costos operacionales aumentan por un $20 \%$, la decisión sería solo tener en operación dos fuentes de retorno y si aumentan por un $30 \%$, solo se opera una fuente de retorno. Además, en todos los casos aumenta la capacidad reservada. Finalmente, cuando los costos fijos de operación aumentan por un $40 \%$, no tiene beneficio el operarlos, por lo que todas las unidades serían adquiridas del proveedor.

\begin{tabular}{|c|c|c|c|}
\hline \multirow[b]{2}{*}{$\alpha$} & \multicolumn{3}{|c|}{$\beta$} \\
\hline & 1 & 0.8 & 0.6 \\
\hline $\mathbf{0}$ & $3(\mathrm{M} / \mathrm{M} / \mathrm{B}) 200$ & \multirow{3}{*}{\multicolumn{2}{|c|}{$3(\mathrm{~A} / \mathrm{A} / \mathrm{M}), 100$}} \\
\hline $10 \%$ & & & \\
\hline $20 \%$ & $2(\mathrm{M} / \mathrm{M} /-), 300$ & & \\
\hline $30 \%$ & $1(-/ \mathrm{M} /-), 400$ & $2(-/ \mathrm{A} / \mathrm{M}), 200$ & \\
\hline $40 \%$ & \multicolumn{2}{|c|}{$0(-/-/-), 500$} & 2 (-/A/M), 200 \\
\hline
\end{tabular}

Tabla XIII.- Decisiones óptimas en los experimentos relacionados a las fuentes de retorno.

4.2. Probabilidad de que no se reciban las unidades requeridas del proveedor.- Se evalúa el efecto de cambios a la probabilidad de que no se reciban las unidades requeridas del proveedor $(w)$. El valor base es $5 \%(w=5 \%)$ y se consideran dos niveles adicionales, una reduccion en la probabilidad, $w=2.5 \%$, y un aumento en la probabilidad, $w=10 \%$.

La Tabla XIV presenta las decisiones para los tres niveles de $w$ bajo la condición base de retornos bajos $(\beta=1)$, y considerando cambios a los costos fijos de operación de las fuentes de retorno: $\alpha=0,20 \%, 40 \%$. Bajo estas condiciones generales hay cinco decisiones óptimas: $3(M / M / B), 200 ; 3(A / A / M), 100 ; 2(M / M /-), 300 ; 2(-/ A / M), 200 ;$ y $0(-/-/-), 500$. En 5 de los 9 puntos de experimentación es óptimo operar tres fuentes de retorno, en dos es óptimo operar dos y en cuatro es óptimo no operar ninguna fuente. Cuando la probabilidad de no recibir es menor, o sea el proveedor y su entorno son más confiables, la decisión base no cambia si los costos operacionales se mantienen al nivel base. En caso de que los costos operacionales aumentan, la decisión es de no utilizar ninguna de las fuentes de retorno. En cambio, si la probabilidad de no recibir las unidades aumenta, como es de esperarse, se reduce la capacidad reservada y se aumentan los incentivos para obtener más unidades usadas.

\begin{tabular}{|c|c|c|c|}
\hline \multirow{2}{*}{$\alpha$} & \multicolumn{3}{|c|}{$w$} \\
\cline { 2 - 3 } & $\mathbf{2 . 5 \%}$ & $\mathbf{5 \%}$ & $\mathbf{1 0 \%}$ \\
\cline { 4 - 4 } $\mathbf{0 \%}$ & \multicolumn{2}{|c|}{$3(M / M / B), 200$} & \multirow{2}{*}{$3(A / A / M), 100$} \\
\hline $\mathbf{2 0 \%}$ & \multirow{2}{*}{$0(-/-/-), 500$} & $2(M / M /-), 300$ & $2(-/ A / M), 200$ \\
& & & $20 \%$ \\
\hline
\end{tabular}

Tabla XIV.- Decisiones óptimas en los experimentos relacionados a $w$ con $\beta=1$.

La Tabla XV presenta las decisiones para los tres niveles de $w$ bajo la condiccion donde la probabilid de retornos bajos se ha reducido por un factor de $0.6(\beta=0.6)$. Bajo estas condiciones solo son óptimas 3 decisiones: $3(A / A / M), 100 ; 2(-/ A / M), 200$; y $0(-/-/-), 500$. En 7 de los 9 puntos de experimentación es óptimo operar 3 fuentes de retorno, en 1 punto es óptimo usar dos fuentes, y en 1 punto es óptimo no operar ninguna fuente. Se puede concluir que en general cuando la probabilidad de retornos bajos es menor, es irrelevante si la probabilidad de recibir las 
unidades del proveedor cambia, se utilizan las tres fuentes de retornos. Solo cuando el costo de operar las fuentes de retorno aumenta por un $40 \%$ y la probabilidad de recibir las unidades es $2.5 \%$ es óptimo no operar ninguna de las fuentes.

\begin{tabular}{|c|c|c|c|}
\hline \multirow[b]{2}{*}{$\alpha$} & \multicolumn{3}{|c|}{$w$} \\
\hline & $2.5 \%$ & $5 \%$ & $10 \%$ \\
\hline $0 \%$ & \multirow{2}{*}{\multicolumn{2}{|c|}{$3(A / A / M), 100$}} & \\
\hline $20 \%$ & & & \\
\hline $40 \%$ & $0(-/-/-), 500$ & $2(-/ A / M), 200$ & \\
\hline
\end{tabular}

Tabla XV.- Decisiones óptimas en los experimentos relacionados a $w$ con $\beta=0.6$.

4.3. Costo por unidad de la demanda no satisfecha.- Se evalúa el costo por unidad de la demanda no satisfecha $\left(c^{\text {perd }}\right)$. El valor base es $\$ 90\left(c^{\text {perd }}=\$ 90\right)$ y se consideran dos niveles adicionales, una reducción en el costo, $c^{\text {perd }}=\$ 45$, y un aumento en el costo, $c^{\text {perd }}=\$ 180$.

La Tabla XVI presenta las decisiones para los tres niveles de $c^{\text {perd }}$ bajo la condiccion base de retornos bajos $(\beta=1)$, y considerando cambios a los costos fijos de operación de las fuentes de retorno: $\alpha=0,20 \%, 40 \%$. Bajo estas condiciones generales hay siete decisiones óptimas: $3(M / M / M), 100 ; 3(M / M / B), 200 ; 3(M / M / M), 200 ; 2(M / M /-), 300 ; 1(-/ A /-), 300$; $1(-/ A /-), 300$ y $0(-/-/-), 500$. En 5 de los 9 puntos de experimentación es óptimo operar tres fuentes de retorno, en uno es óptimo operar dos, en dos puntos es óptimo operar un fuente, y en un punto es óptimo no operar ninguna fuente. El cuadro en gris representa el caso base. En este conjunto de experimentos se observan varios efectos interesantes. Cuando $\alpha=0$ y $c^{\text {perd }}=\$ 45$, la decisión es $3(M / M / M), 100$; mientras que cuando $c^{\text {perd }}=\$ 180$, son las mismas fuentes y los mismos incentivos, pero se reserva al próximo nivel de capacidad $(3(M / M / M), 200)$. Cuando $\alpha=40 \%$ y $c^{\text {perd }}=\$ 45$ se utiliza solo la fuente $f 2$ y se reserva una capacidad de 300 , cuando $c^{\text {perd }}=\$ 90$ no se opera ninguna fuente, mientras que cuando $c^{\text {perd }}=\$ 180$, se usa la fuente $f 2$ con el incentivo Bajo, pero la capacidad reservada aumenta en comparación a $c^{\text {perd }}=\$ 45$.

\begin{tabular}{|c|c|c|c|}
\hline \multirow{2}{*}{$\alpha$} & \multicolumn{3}{|c|}{$c^{\text {perd }}$} \\
\cline { 3 - 3 } & $\mathbf{\$ 4 5}$ & $\mathbf{\$ 9 0}$ & $\mathbf{\$ 1 8 0}$ \\
\cline { 1 - 1 } $\mathbf{0 \%}$ & $3(M / M / M), 100$ & $3(\mathrm{M} / \mathrm{M} / \mathrm{B}), 200$ & \multirow{2}{*}{$3(\mathrm{M} / \mathrm{M} / \mathrm{M}), 200$} \\
\cline { 1 - 1 } $\mathbf{2 0 \%}$ & $1(-/ \mathrm{A} /-), 300$ & $2(\mathrm{M} / \mathrm{M} /-), 300$ & $1(-/ \mathrm{M} /-), 400$ \\
\hline $\mathbf{4 0 \%}$ & $0(-/-), 500$ & \\
\hline
\end{tabular}

Tabla XVI.- Decisiones óptimas en los experimentos relacionados a ct $^{\text {perd }}$ con $\beta=1$.

La Tabla XVII presenta las decisiones para los tres niveles de $w$ bajo la condiccion donde la probabilid de retornos bajos se ha reducido por un factor de $0.6(\beta=0.6)$. Bajo estas condiciones generales hay cinco decisiones óptimas: $3(M / B / M), 100 ; 3(A / A / M), 100 ; 3(A / A /$ $A), 100 ; 2(-/ A / M), 200 ; y 1(-/ A /-), 300$. En 7 de los 9 puntos de experimentación es óptimo operar tres fuentes de retorno, en un punto es óptimo operar dos, y en un punto experimental es óptimo operar una fuente. En estos experimentos nunca fue óptimo el no operar ninguna fuente. También se observa que en este conjunto de experimentos mientras $c^{\text {perd }}$ aumenta, aumentan los incentivos hasta el punto donde se da el nivel de incentivo Alto a todas las fuentes cuando $c^{\text {perd }}=$ $\$ 180$. 


\begin{tabular}{|c|c|c|c|}
\hline \multirow{2}{*}{$\alpha$} & \multicolumn{3}{|c|}{$c^{\text {perd }}$} \\
\cline { 2 - 3 } $\mathbf{0 \%}$ & $\mathbf{\$ 4 5}$ & $\mathbf{\$ 9 0}$ & $\mathbf{\$ 1 8 0}$ \\
\cline { 1 - 1 } $\mathbf{2 0 \%}$ & $3(\mathrm{M} / \mathrm{B} / \mathrm{M}), 100$ & $3(\mathrm{~A} / \mathrm{A} / \mathrm{M}), 100$ & \multirow{2}{*}{$3(\mathrm{~A} / \mathrm{A} / \mathrm{A}), 100$} \\
\hline $\mathbf{4 0 \%}$ & $1(-/ \mathrm{A} /-), 300$ & $2(-/ \mathrm{A} / \mathrm{M}), 200$ & \\
\hline
\end{tabular}

Tabla XVII. - Decisiones óptimas en los experimentos relacionados a ct $^{\text {perd }}$ con $\beta=0.6$.

Los experimentos realizados demuestran que el número de fuentes de retorno, cuáles en particular se utilizan, y los incentivos que se otorgarán a cada fuente depende de las cuatro variables analizadas y las posibles interacciones entre las variables. Como es de esperarse, mientras mayor sea la probabilidad de altos niveles de retorno, más fuentes estarán en operación y más incentivos se les dará, y por consiguiente menos capacidad se reserva. Si los costos de operar las fuentes aumentan, lo opuesto ocurre, menos fuentes y más capacidad se reserva. En esos casos el nivel de incentivos aumenta. Cuando la probabilidad de recibir las unidades requeridas del proveedor aumenta, se prefiere reservar capacidad, pero dependiendo si los costos de operación son los bases o si son mayores. Finalmente, en relación con los costos asociados con la demanda no satisfecha, mientras mayor sean estos por unidad, más fuentes estarán en operación y más incentivos se ofrecerán. El modelo claramente demuestra la complejidad del problema y como la decisión óptima depende de los parámetros y condiciones propios del sistema.

5. Conclusiones y trabajos futuros.- Este trabajo contribuye a los conocimientos sobre CSCC con un modelo que considera múltiples variables importantes en un sistema de remanufactura. Este tipo de modelo es relevante para la comunidad científica y profesional dada la creciente preocupación por el medio ambiente, las progresivas regulaciones ambientales y la renovada importancia de la responsabilidad social corporativa. En adición, más y más empresas se han dado cuenta que el diseño eficaz de redes de recuperación de productos puede resultar en una ventaja competitiva (además del creciente interés por conseguir una manufactura más sustentable).

Dada la alta variabilidad que existe actualmente en las CS en cuanto a la cantidad de los productos retornados, sumado al hecho de que los requisitos de producción generalmente superan las unidades usadas entrantes, obliga a las cadenas de suministro de ciclo cerrado a depender de una mezcla de componentes nuevos y remanufacturados. Las empresas pueden proveer incentivos a los usuarios de productos y así promover el reemplazo de sus unidades usadas por unidades nuevas. El proveer incentivos puede resultar en mayores volúmenes de retornos y retornos en mejores condiciones. Pero esto implica costos adicionales que tienen que ser balanceados con el costo de nuevas unidades. El modelo que presenta esta investigación resulta de principal interés para el mundo empresarial, en cuanto permite conseguir la solución óptima que "balancea" todos los elementos de costos.

Hay múltiples direcciones para investigaciones relacionadas con los sistemas de remanufactura donde se utilizan incentivos y se reserva capacidad. Una de estas consistiría en incluir en el modelo la duración del ciclo de reemplazo, lo cual determinaría los niveles de inventario de los componentes, y los costos de procesar las órdenes y los centros de retorno. Otra área interesante sería incluir un elemento de riesgo asociado con los retornos, donde estos no sean utilizables por razones de calidad o tiempo de recibo. En la práctica, es muy importante desarrollar modelos y tecnologías que apoyen conseguir fuentes de retorno confiables y constantes, con volúmenes que garantizan la sustentabilidad y la rentabilidad del proceso. 


\section{Referencias}

[1] Govindan, K., Soleimani, H., 2017. A review of reverse logistics and closed-loop supply chains: a Journal of Cleaner Production focus. Journal of cleaner production 142, 371384. https://doi.org/10.1016/j.jclepro.2016.03.126

[2] Pishvaee, M. S., Jolai, F., Razmi, J., 2009. A stochastic optimization model for integrated forward/reverse logistics network design. Journal of Manufacturing Systems. 28(4), 107114. https://doi.org/10.1016/j.jmsy.2010.05.001

[3] Zeballos, L. J., Méndez, C. A., Barbosa-Povoa, A. P., 2016. Design and planning of closed-loop supply chains: A risk-averse multistage stochastic approach. Industrial \& Engineering Chemistry Research. 55(21), 6236-6249. http://dx.doi.org/10.1021/acs.iecr.5b03647

[4] Coenen, J., Van der Heijden, R. E., van Riel, A. C., 2018. Understanding approaches to complexity and uncertainty in closed-loop supply chain management: Past findings and future directions. Journal of cleaner production 201, 1-13. https://doi.org/10.1016/j.jclepro.2018.07.216

[5] Qin, Z., Ji, X., 2010. Logistics network design for product recovery in fuzzy environment. Eur. J. of Op. Res. 202(2), 479-490. https://doi.org/10.1016/j.ejor.2009.05.036

[6] Agrawal, S., Singh, R. K., Murtaza, Q., 2015. A literature review and perspectives in reverse logistics. Resources, Conservation and Recycling 97, 7692.https://doi.org/10.1016/j.resconrec.2015.02.009

[7] Masoudipour, E., Amirian, H., Sahraeian, R., 2017. A novel closed-loop supply chain based on the quality of returned products. Journal of cleaner production. 151, 344-355. https://doi.org/10.1016/j.jclepro.2017.03.067

[8] Maiti, T., Giri, B. C., 2015. A closed loop supply chain under retail price and product quality dependent demand. J. of Manuf. Syst. 37, 624-637. https://doi.org/10.1016/j.jmsy.2014.09.009

[9] Liu, L., Wang, Z., Xu, L., Hong, X., \& Govindan, K., 2017. Collection effort and reverse channel choices in a closed-loop supply chain. Journal of cleaner production 144, 492500. https://doi.org/10.1016/j.jclepro.2016.12.126

[10] Taleizadeh, A. A., Haghighi, F., Niaki, S. T. A., 2019. Modeling and solving a sustainable closed loop supply chain problem with pricing decisions and discounts on returned products. Journal of cleaner production. 207, 163-181. https://doi.org/10.1016/j.jclepro.2018.09.198.

[11] Tanco, M., Jurburg, D., \& Escuder, M. (2015). Main difficulties hindering supply chain performance: an exploratory analysis at Uruguayan SMEs. Supply Chain Management: An International Journal, 20(1), 11-23. 
[12] Klausner, M., \& Hendrickson, C. T. (2000). Reverse-logistics strategy for product takeback. Interfaces, 30(3), 156-165.

https://doi.org/10.1287/inte.30.3.156.11657

[13] Heese, H. S., Cattani, K., Ferrer, G., Gilland, W., Roth, A. V., 2005. Competitive advantage through take-back of used products. Eur. J. of Op. Res., 164(1), 143-157. https://doi.org/10.1016/j.ejor.2003.11.008

[14] Matsumoto, M., Umeda, Y., 2011. An analysis of remanufacturing practices in Japan. Journal of Remanufacturing. 1(1), 2. 10.1186/2210-4690-1-2

[15] Atasu, A., Sarvary, M., \& Van Wassenhove, L. N. (2008). Remanufacturing as a marketing strategy. Management science, 54(10), 1731-1746.

https://doi.org/10.1287/mnsc. 1080.0893

[16] De Brito, M. P., Dekker, R., \& Flapper, S. D. P. (2005). Reverse logistics: a review of case studies. In Distribution Logistics (pp. 243-281). Springer, Berlin, Heidelberg.

[17] Ruiz-Torres, A.J., Mahmoodi, F., Ohmori, S., 2019. Joint determination of supplier capacity and returner incentives in a closed-loop supply chain. Journal of cleaner production. 215, 1351-1361.

[18] Govindan, K., Soleimani, H., Kannan, D., 2015. Reverse logistics and closed-loop supply chain: A comprehensive review to explore the future. European journal of operational research 240(3), 603-626. https://doi.org/10.1016/j.ejor.2014.07.012

[19] Braz, A.C., De Mello, A.M., de Vasconcelos Gomes, L.A., de Souza Nascimento, P.T., 2018. The bullwhip effect in closed-loop supply chains: A systematic literature review. $\begin{array}{llll}\text { Journal of } & \text { cleaner } & \text { 376-389. }\end{array}$ https://doi.org/10.1016/j.jclepro.2018.08.042

[20] De Giovanni, P., \& Zaccour, G. (2019). A selective survey of game-theoretic models of closed-loop supply chains. 4OR, 17(1), 1-44. https://doi.org/10.1007/s1028

[21] El-Sayed, M., Afia, N., El-Kharbotly, A., 2010. A stochastic model for forward-reverse logistics network design under risk. Computers \& Industrial Engineering 58(3), 423-431. https://doi.org/10.1016/j.cie.2008.09.040

[22] Zeballos, L. J., Gomes, M. I., Barbosa-Povoa, A. P., Novais, A. Q., 2012. Addressing the uncertain quality and quantity of returns in closed-loop supply chains. Computers \& Chemical Engineering. 47, https://doi.org/10.1016/j.compchemeng.2012.06.034

237-247.

[23] Benedito, E., Corominas, A., 2013. Optimal manufacturing policy in a reverse logistic system with dependent stochastic returns and limited capacities. International Journal of Production Research 51(1), 189-201.https://doi.org/10.1080/00207543.2012.655863 
[24] Cardoso, S. R., Barbosa-Póvoa, A. P. F., Relvas, S., 2013. Design and planning of supply chains with integration of reverse logistics activities under demand uncertainty. European Journal of Operational Research 226(3), 436-451. https://doi.org/10.1016/j.ejor.2012.11.035

[25] Zeballos, L. J., Méndez, C. A., Barbosa-Povoa, A. P., Novais, A. Q., 2014. Multi-period design and planning of closed-loop supply chains with uncertain supply and demand. Computers \& Chemical Engineering. 66, 151-164. https://doi.org/10.1016/j.compchemeng.2014.02.027

[26] Khatami, M., Mahootchi, M., Farahani, R. Z., 2015. Benders' decomposition for concurrent redesign of forward and closed-loop supply chain network with demand and return uncertainties. Transportation Research Part E: Logistics and Transportation Review 79, 1-21. https://doi.org/10.1016/j.tre.2015.03.003

[27] Giri, B. C., \& Sharma, S., 2016. Optimal production policy for a closed-loop hybrid system with uncertain demand and return under supply disruption. Journal of cleaner production 112, 2015-2028. https://doi.org/10.1016/j.jclepro.2015.06.147

[28] Keyvanshokooh, E., Ryan, S. M., Kabir, E., 2016. Hybrid robust and stochastic optimization for closed-loop supply chain network design using accelerated Benders decomposition. European journal of operational research 249(1), 76-92. https://doi.org/10.1016/j.ejor.2015.08.028

[29] Hosoda, T., S. M. Disney., 2018. A unified theory of the dynamics of closed-loop supply chains. European journal of operational research 269(1), 313-326. https://doi.org/10.1016/j.ejor.2017.07.020

[30] Zeballos, L.J., Méndez, C.A. and Barbosa-Povoa, A.P., 2018. Integrating decisions of product and closed-loop supply chain design under uncertain return flows. Computers \& Chemical $\quad$ Engineering. 211-238. https://doi.org/10.1016/j.compchemeng.2018.02.011

[31] Wu, G.H., Chang, C.K. and Hsu, L.M., 2018. Comparisons of Interactive Fuzzy Programming Approaches for Closed-loop Supply Chain Network Design under Uncertainty. Computers \& Industrial Engineering 68. https://doi.org/10.1016/j.cie.2018.09.022.

[32] Kim, J., Do Chung, B., Kang, Y., Jeong, B., 2018. Robust optimization model for closedloop supply chain planning under reverse logistics flow and demand uncertainty. Journal of cleaner production 196, 1314-1328. https://doi.org/10.1016/j.jclepro.2018.06.157

[33] Ponte, B., M.M. Naim, A. A. Syntetos. (Forthcoming). The effect of returns volume uncertainty on the dynamic performance of closed-loop supply chains. Journal of Remanufacturing (2019). https://doi.org/10.1007/s13243-019-00070-x

[34] Aras, N., Aksen, D., 2008. Locating collection centers for distance-and incentivedependent returns. International Journal of Production Economics 111(2), 316-333. https://doi.org/10.1016/j.ijpe.2007.01.015 
[35] Aras, N., Aksen, D., Tanuğur, A. G., 2008. Locating collection centers for incentivedependent returns under a pick-up policy with capacitated vehicles. European Journal of Operational Research 191(3), 1223-1240. https://doi.org/10.1016/j.ejor.2007.08.002

[36] He, Y., 2015. Acquisition pricing and remanufacturing decisions in a closed-loop supply chain. International Journal of Production Economics 163, 48-60. https://doi.org/10.1016/j.ijpe.2015.02.002

[37] De Giovanni, P., Reddy, P. V., Zaccour, G., 2016. Incentive strategies for an optimal recovery program in a closed-loop supply chain. European Journal of Operational Research 249(2), 605-617. https://doi.org/10.1016/j.ejor.2015.09.021

[38] Bhattacharya, R., Kaur, A., \& Amit, R. K., 2018. Price optimization of multi-stage remanufacturing in a closed loop supply chain. Journal of cleaner production 186, 943 962. https://doi.org/10.1016/j.jclepro.2018.02.222

[39] Modak, N. M., Modak, N., Panda, S., Sana, S. S., 2018. Analyzing structure of twoechelon closed-loop supply chain for pricing, quality and recycling management. Journal of cleaner production. 171, 512-528. https://doi.org/10.1016/j.jclepro.2017.10.033

[40] Aissaoui N, Haouari M, Hassini E. 2007, Supplier selection and order lot sizing modeling: A review. Computers \& operations research 34, 3516-3540.

[41] Ho, W., Xu, X., Dey, P.K., 2010. Multi-criteria decision-making approaches for supplier evaluation and selection: A literature review. European journal of operational research 202, 16-24.

[42] Sawik, T., 2013, Selection of resilient supply portfolio under disruption risks. Omega. 41, 259-269.

[43] Berger, P.D., Gerstenfeld, A., Zeng, A.Z., 2004, How many suppliers are best? A decision making approach. Omega. 32(1), 9-15.

[44] Ruiz-Torres, A.J., Mahmoodi, F., 2006, A supplier allocation model considering delivery failure, maintenance and supplier cycle costs. International Journal of Production Economics. 103(2), 755-766.

[45] Moritz, S., \& Pibernik, R. (2008). The optimal number of suppliers in the presence of volume discounts and different compensation potentials-an analytical and numerical analysis. European Business School Research Paper, (0903). https://dx.doi.org/10.2139/ssrn.1358037

[46] Sarkar A., Mohapatra, P.K.J., 2009, Determining the optimal size of supply base with the consideration of risks of supply disruptions. International Journal of Production Economics. 119(1), 122-135.

[47] Meena, P.L., Sarmah, S.P., Sarkar, A., 2011. Sourcing decisions under risks of catastrophic event disruptions. Transportation Research Part E: Logistics and Transportation Review. 47(6), 1058-1074. 
[48] Sawik, T., 2011, Supplier selection in make-to-order environment with risks. Mathematical and Computer Modelling. 53(9-10), 1670-1679.

[49] Meena, P.L., Sarmah, S.P., 2013, Multiple sourcing under supplier failure risk and quantity discount: A genetic algorithm approach. Transportation Research Part E: Logistics and Transportation Review. 50 (C), 84-97.

[50] Ruiz-Torres, A.J., Mahmoodi, F., Zeng, A.Z., 2013, Supplier selection model with contingency planning for supplier failures. Computers \& Industrial Engineering. 66(2), 374-382.

[51] Torabi, S.A., Baghersad, M., Mansouri, S.A., 2015, Resilient supplier selection and order allocation under operational and disruption risks. Transportation Research Part E: Logistics and Transportation Review. 79, 22-48.

[52] Kamalahmadi, M., \& Mellat-Parast, M. (2016). Developing a resilient supply chain through supplier flexibility and reliability assessment. International Journal of Production Research, 54(1), 302-321. https://doi.org/10.1080/00207543.2015.1088971

[53] Meena, P.L., Sarmah, S.P., 2016, Supplier selection and demand allocation under supply disruption risks The International Journal of Advanced Manufacturing Technology 83, 265-274.

[54] Sawik, T., 2018, Selection of a dynamic supply portfolio under delay and disruption risks. International Journal of Production Research. 56(1-2), 760-782. https://www.tandfonline.com/doi/full/10.1080/00207543.2017.1401238

[55] Esmaeili-Najafabadi, E., Nezhad, M. S. F., Pourmohammadi, H., Honarvar, M., \& Vahdatzad, M. A. (2019). A joint supplier selection and order allocation model with disruption risks in centralized supply chain. Computers \& Industrial Engineering 127, 734-748. https://doi.org/10.1016/j.cie.2018.11.017

[56] Lücker, F., Seifert, R. W., \& Biçer, I. (2019). Roles of inventory and reserve capacity in mitigating supply chain disruption risk. European Journal of Operational Research 57(4), 1238-1249. https://doi.org/10.1080/00207543.2018.1504173

[57] Hosseini, S., Morshedlou, N., Ivanov, D., Sarder, M.D., Barker, K, Al Khaled, A., 2019. Resilient supplier selection and optimal order allocation under disruption risks. International Journal of Production Economics. 213, 124-137. 